\title{
Detection of Defect Edge of Aluminum Foils Based on Fuzzy Enhancement and Wavelet Transform
}

\author{
Hui Wang ${ }^{1, *}$, Huanhuan $\mathrm{Li}^{2}$,Yongfa Ling ${ }^{1}$, Hongyan Zhang ${ }^{1}$ and Xiangxu Xie ${ }^{1}$ \\ ${ }^{1}$ School of Mechanical and Electronic Engineering, Hezhou University, Hezhou 542899, China \\ ${ }^{2}$ Jiangxi Vocational College of Mechanical \& Electrical Technology, Nanchang 330013, China \\ ${ }^{*}$ Corresponding author
}

\begin{abstract}
In order to accurately detect the defects on the aluminum surface, including pinholes, yellow discoloration, oil stains, and scratches, an algorithm combining local fuzzy enhancement, wavelet transform modulus maxima (WTMM) and multi-scale product was proposed. First, the defect areas with low contrast, such as yellow discoloration and oil stains, were located in the HSI color space; then, the local fuzzy enhancement was performed on these edges, thereby highlighting the edge features and reducing the amount of computation; at last, the multi-scale product was calculated, and the binary image of defect edge on aluminum foil was obtained via WTMM. Plenty of experimental results showed that the proposed algorithm could extract clearer and more complete edges and effectively detect the defect edges with low contrast in the aluminum foil image, laying the foundation for the subsequent defect identification.
\end{abstract}

Keywords-edge detection; wavelet transform; modulus maxima; fuzzy enhancement; multi-scale product

\section{INTRODUCTION}

As a raw material of industrial manufacturing and daily life, aluminum foil is widely used in the fields such as electronics, packaging, and construction. However, since some defects may occur in its production process due to the impacts of production equipment and techniques, the accurate detection of these defects on the aluminum foil surface will directly influence its value in use. The conventional detection of defects on aluminum foil surface basically depends on visual inspection, which is susceptible to subjective senses of the inspector and thus inefficient. The method based on machine vision ${ }^{[1]}$ has high detection efficiency and strong adaptability to the working conditions, gradually becoming the mainstream method of nondestructive examination of product defects. As extracting the image's edge information is an important part of image analysis through the machine vision, a lot of edge detection algorithms have been applied in many fields of visual detection. LIN Li-jun ${ }^{[2]}$ combined the image's weighted information entropy and wavelet transform modulus maxima (WTMM) and applied this approach to detect the defect edge of magnetic tile cracks, achieving the desired effect in the continuity and locating accuracy of the crack defect edge. ZHAO Jiu-liang ${ }^{[3]}$ used the WTMM and multi-scale approach to effectively locate the defect edges on the surface of cold rolled strip, obtaining good results in detection accuracy. ZHANG Xue-wu ${ }^{[4]}$ adopted the method based on vision bionics to inspect the surface defects of copper strips. Based on deep learning, WANG Xian-bao ${ }^{[5]}$ detect the defect edges on the surface of solar cells, with certain advantages in terms of speed. GENG Xin ${ }^{[6]}$ employed a physical model to detect the edges in color image, largely overcoming the false edges introduced by highlights and shadows. HUANG Hai-long ${ }^{[7]}$ combined image local enhancement and multiplex order morphology in edge detection, showing good robustness to noises and different formats of images. Because of the characteristics of its own production process, the edge detection of aluminum foil has been rarely investigated.

In this paper, an algorithm combining local fuzzy enhancement, WTMM and multi-scale product was proposed to detect the defect edges on aluminum foil surface. The local fuzzy enhancement was performed on the defect areas with low contrast to highlight the features of defect edge; and on this basis, the algorithm combining WTMM and multi-scale product was used to detect the defect edge, obtaining the corresponding binary image. On the basis of the isotropy of the two-dimensional wavelet, the WTMM method provided the anti-noise property for the edge detection. Meanwhile, the multi-scale product was calculated, the wavelet transform coefficients in adjacent scales were multiplied to enhance the edge coefficients and reduce the background texture coefficients of the image. Results showed that the algorithm was of significant practical value, as it could not only effectively detect the edge information of pinholes on aluminum foil surface, but also accurately detect the defect edges with low contrast, such as oil stains, yellow discoloration, and scratches.

\section{KEY CONCEPTS}

\section{A. Fuzzy Enhancement}

Image enhancement refers to the process of highlighting some features of interest in an image while reducing the unnecessary information to improve the image's visual effects, thus facilitating further analysis by human or machine. In recent years, the fuzzy set theory ${ }^{[8-9]}$ has been widely researched and applied in the field of image, achieving better processing results than the conventional methods. Similar to the image enhancement in the spatial domain, in the fuzzy feature domain, the image's contrast can be enhanced by modifying the pixels. The image enhancement based on fuzzy set is achieved by three steps: extraction of fuzzy features, modification of membership function values, and inverse transformation of fuzzy domain.

First, and image could be transformed from the spatial domain to the fuzzy domain as follows: 


$$
\mu_{m n}=G\left(g_{m n}\right)=\left[1+\frac{g_{\max }-g_{m n}}{F_{d}}\right]^{-F_{e}}
$$

where $F_{d}$ and $F_{e}$ denote the fuzzy parameters, $g_{\max }$ denotes the maximum grayscale value in the image, and $g_{m n}$ denotes the grayscale of the current pixel.

$$
T\left(\mu_{m n}\right)= \begin{cases}2 \cdot\left[\mu_{m n}\right]^{2} & 0 \leq \mu_{m n} \leq 0.5 \\ 1-2 \cdot\left[1-\mu_{m n}\right]^{2} & 0.5<\mu_{m n} \leq 1\end{cases}
$$

Then, the fuzzy enhancement operator was called through regression to modify the degree of membership. The result of the above formula was to increase $\mu_{m n}$ (when $\mu_{m n}>0.5$ ) or

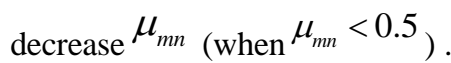

At last, a new gray level was generated through the inverse transform of fuzzy domain, converting the image data from the fuzzy domain to the spatial domain.

$$
g_{m n}{ }^{\prime}=G^{-1}\left(\mu_{m n}{ }^{\prime}\right)=g_{m n}-F_{d}\left[\left(\mu_{m n}\right)^{\frac{-1}{F_{e}}}-1\right]
$$

\section{B. WTMM and Multi-scale Product}

Edge detection requires the ability to not only accurately detect the target edge, but also inhibit the interference of background texture. Since the single-scale edge detection can hardly meet the above requirements, the multi-scale approach turns out to be a good way to achieve the effective edge information. By calculating the multi-scale product ${ }^{[10-11]}$, the wavelet transform coefficients in adjacent scales are multiplied to enhance the edge coefficients and reduce the noises. However, due to the strong correlation between the scale space on the image's edge, when the wavelet coefficients in adjacent scale are multiplied, the results will contain the edge information between different scales. After that, threshold process with local modulus maxima ${ }^{[12]}$ was performed on the wavelet transform coefficients to obtain the image's edge information.

Let $\theta(x, y)$ be an appropriate two-dimensional smooth function, and the following conditions are met:

$$
\begin{aligned}
& \int_{-\infty}^{+\infty} \int_{-\infty}^{+\infty} \theta(x, y)=1 \\
& \lim _{x^{2}+y^{2} \rightarrow \infty} \theta(x, y) \rightarrow 0
\end{aligned}
$$

When the scale was $s$, assume:

$$
\theta_{s}(x, y)=\frac{1}{s^{2}} \theta\left(\frac{x}{s}, \frac{y}{s}\right)
$$

then, in the scale $S$, the 2 two-dimensional wavelet functions were defined as:

$$
\begin{aligned}
& \psi_{s}^{x}(x, y)=\frac{\partial \theta_{s}(x, y)}{\partial x}=\frac{1}{s^{2}} \psi^{x}\left[\frac{x}{s}, \frac{y}{s}\right] \\
& \psi_{s}^{y}(x, y)=\frac{\partial \theta_{s}(x, y)}{\partial y}=\frac{1}{s^{2}} \psi^{y}\left[\frac{x}{s}, \frac{y}{s}\right]
\end{aligned}
$$

When scale $S=2^{j}$, the 2 two-dimensional dyadic wavelet transform components of image $f(x, y)$ were:

$$
\left[\begin{array}{l}
\omega_{2^{j}}^{x} f(x, y) \\
\omega_{2^{j}}^{y} f(x, y)
\end{array}\right]=\left[\begin{array}{l}
\frac{\partial}{\partial x}\left(f^{*} \theta_{2^{j}}\right)(x, y) \\
\frac{\partial}{\partial y}\left(f^{*} \theta_{2^{j}}\right)(x, y)
\end{array}\right]=2^{j} \vec{\nabla}\left(f^{*} \theta_{2^{j}}\right)(x, y)
$$

where $\omega_{2^{j}}^{x} f(x, y)$ and $\omega_{2^{j}}^{y} f(x, y)$ respectively denotes the 2 gradient vector components of image $f(x, y)$, in the horizontal and vertical directions after processing by the smooth function $\theta_{s}(x, y)$. The corresponding normalized scale product was:

$$
\begin{aligned}
& P_{2^{j}}^{x} \mathrm{f}(x, y)=\frac{\omega_{2^{j}}^{x} \mathrm{f}(x, y) \cdot \omega_{2^{j+1}}^{x} \mathrm{f}(x, y)}{\sqrt{\omega_{2^{j}}^{x} \mathrm{f}(x, y) \cdot \omega_{2^{j+1}}^{x} \mathrm{f}(x, y)}} \\
& P_{2^{j}}^{y} \mathrm{f}(x, y)=\frac{\omega_{2^{j}}^{y} \mathrm{f}(x, y) \cdot \omega_{2^{j+1}}^{y} \mathrm{f}(x, y)}{\sqrt{\omega_{2^{j}}^{y} \mathrm{f}(x, y) \cdot \omega_{2^{j+1}}^{y} \mathrm{f}(x, y)}}
\end{aligned}
$$

The product modulus and direction of the wavelet transform of gradient vector $\vec{\nabla}\left(\mathrm{f}^{*} \theta_{2^{j}}\right)(x, y)$ in the adjacent scale were:

$$
\begin{array}{r}
M_{2}^{j} \mathrm{f}(x, y)=\sqrt{\left|P_{2^{j}}^{x} \mathrm{f}(x, y)\right|^{2}+\left|P_{2^{j}}^{y} \mathrm{f}(x, y)\right|^{2}} \\
A_{2}^{j} \mathrm{f}(x, y)=\arctan \frac{P_{2^{j}}^{y} \mathrm{f}(x, y)}{P_{2^{j}}^{x} \mathrm{f}(x, y)}
\end{array}
$$

The local maxima of $M_{2}^{j} \mathrm{f}(x, y)$ on the direction $A_{2}^{j} \mathrm{f}(x, y)$ of the gradient vector was the edge feature point of image $\mathrm{f}(x, y)$.

\section{DESIGN AND IMPLEMENTATION OF ALGORITHM}

\section{A. Local Fuzzy Enhancement}

The local fuzzy enhancement can effectively highlight the image edge features and simultaneously reduce the amount of computation. In addition, determining the area to be enhanced is the key of local fuzzy enhancement. For defects with low contrast, such as yellow discoloration and oil stains, it is 
difficult to distinguish the defects from the background texture by the brightness. Therefore, a reliable model should be established to detect these defect edges. The color space HSI (hue, saturation, and intensity) model can separate the hue information from the intensity component embedded in the image. As the components $\mathrm{H}$ and $\mathrm{S}$ contain important color information, the color can be converted from RGB to HSI by the following equation:

$$
H=\left\{\begin{array}{cc}
\theta & B \leq G \\
360-\theta & B>G
\end{array}\right.
$$

where

$$
\begin{gathered}
\theta=\cos ^{-1}\left\{\frac{0.5[(R-G)+(R-B)]}{\left[(R-G)^{2}+(R-B)(G-B)\right]^{1 / 2}}\right\} \\
S=1-\frac{3}{(R+G+B)}[\min (R, G, B)] \\
I=\frac{1}{3}(R, G, B)
\end{gathered}
$$

Experiments were carried out to analyze a lot of defect sample, such as yellow discoloration and oil stains. Results indicated that these defects showed good clustering feature in the $S$ (saturation) component, with their statistical distribution as: $0.2<s<0.3$. These defect models were located through conversion into binary images. Then the fuzzy enhancement was performed in the local area of the defect.

\section{B. Detection of Defect Edge Based on WTMM and Multi- scale Product}

For a defect image of aluminum foil, on the basis of local fuzzy enhancement, WTMM and multi-scale product were adopted to segment the targets, including pinholes, oil stains and yellow discoloration. The specific steps are as follows:

Step 1: A dyadic discrete stationary wavelet transform was performed for the original aluminum foil image;

Step 2: The horizontal and vertical transform coefficients of the aluminum foil image were obtained via the wavelet transform coefficients;

Step 3: The scale products of the horizontal and vertical wavelet transform coefficients were calculated respectively;

Step 4: The wavelet transform modulus and gradient direction were calculated;

Step 5: The corresponding local modulus maxima in the gradient direction was calculated, that is, the edge points of the image.

\section{Steps and Processes of Algorithm}

In order to improve the accuracy of edge detection and reduce the amount of computation, the fuzzy enhancement was only performed in the defect areas with low contrast. In this paper, an algorithm combining local fuzzy enhancement,
WTMM and multi-scale product was proposed to detect the surface defect of aluminum foil. The steps of the algorithm were detailed as follows:

Step 1: A color image of aluminum foil was converted into HSI color space, in which the defects like yellow discoloration and oil stains showed good clustering feature in the $\mathrm{S}$ component;

Step 2: After obtaining the binary image, the central coordinate positions of the defects were further calculated;

Step 3: The defect areas with low contrast were located. Since the surface defects of aluminum foil were irregularly and discontinuously distributed, results calculated in Step 2 might lead to multiple closely spaced coordinates in a small area. For this reason, the detection results should be merged, so that the fuzzy enhancement could be performed in only one area, thereby reducing the amount of computation. In merging areas, this paper employed a K-means clustering method based on distance (dist), in which dist was empirical value. In this paper, through a large number of experiments, dist was set to 30 .

Step 4: The local fuzzy enhancement was performed in the area of defects;

Step 5: The scale product of the wavelet transform coefficient in adjacent scales was calculated, and the binary image of the defect edge was obtained through WTMM.

The overall process of the algorithm is shown in Fig.1:

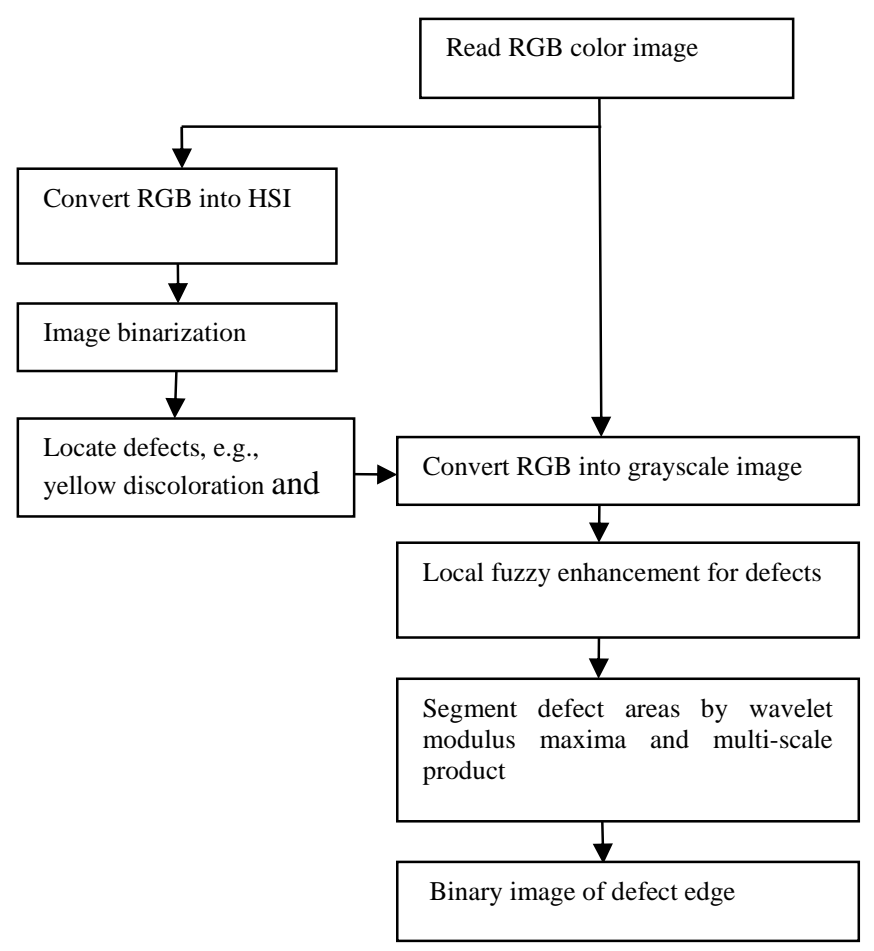

FIGURE I. FLOWCHART OF THE PROPOSED METHOD

\section{RESUlts AND DisCUSSION}

In this study, three images showing various defects on the surface of aluminum foil were selected for analysis. Fig.2 (a) shows the pinholes, yellow discoloration and oil stains on 
aluminum foil; Fig.2(b) the pinholes, oil stains and scratches; and Fig.2(c) the pinholes. During the experiments, all the detection was conducted by the algorithm combining wavelet modulus maxima and multi-scale product. The results of direct direction on the 3 selected images were shown in Fig.3; first, the fuzzy enhancement was conducted on the whole image, then the defect edges were detected, with results as shown in Fig.4; in the proposed method, the fuzzy enhancement was first performed in local areas, then the defect edges were detected, with results as shown in Fig.5.

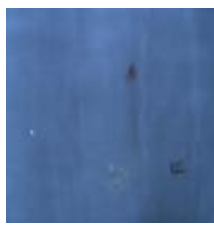

(a)

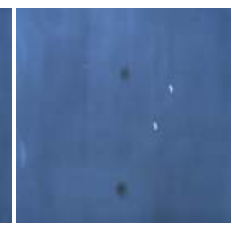

(b)

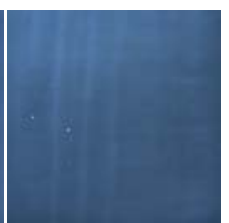

(c)
FIGURE II. ORIGINAL IMAGES OF DEFECTS ON ALUMINUM FOIL SURFACE

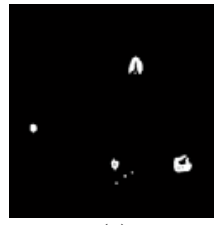

(a)

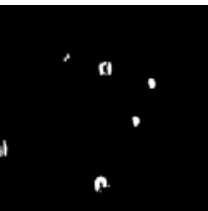

(b)

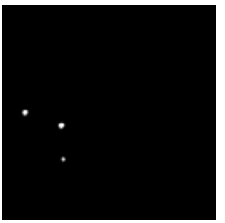

(c)

FIGURE III. DETECTION RESULTS OF DEFECT EDGES BY WAVELET MODULUS MAXIMA AND MULTI-SCALE PRODUCT

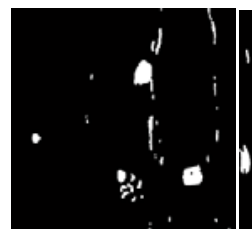

(a)

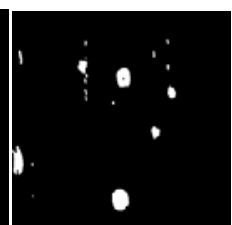

(b)

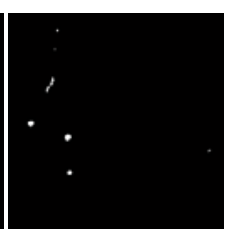

(c)
FIGURE IV. DETECTION RESULTS OF DEFECT EDGES IN THE WHOLE IMAGE AFTER FUZZY ENHANCEMENT

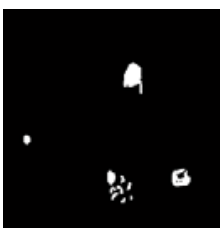

(a)

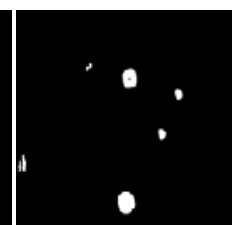

(b)

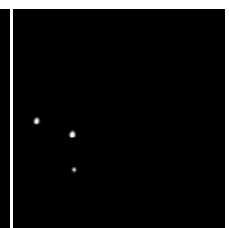

(c)
FIGURE V. DETECTION RESULTS OF DEFECT EDGES BY THE PROPOSED METHOD

Fig.3 to 5 show the results of detection by different methods. According to Fig.3(a), some yellow discoloration was undetected, and the oil stains were incompletely detected; in Fig.3(b), the oil stains were incompletely detected; in Fig.3(c), the edges of pinholes were clearly defected. According to Fig.4, the defect areas were detected along with some non-defect background texture, which might interfere with the subsequent defect identification and make it difficult to extract the defects on aluminum foil surface, including pinholes, yellow discoloration, oil stains, and scratches. The results of the proposed method were shown in Fig.5, and all these defects could be accurately detected, with clearer and more complete edges.

A total of 150 images of aluminum foil were selected in this study. Among them, 80 showed no surface defect, while 70 showed defects including pinholes, yellow discoloration, oil stains and scratches (some showed a single kind of defect, and the others showed multiple ones). The results of the proposed detection method were shown in Table 1:

TABLE I. DETECTION RESULTS OF DEFECT EDGES

\begin{tabular}{|c|c|c|c|}
\hline Samples & $\begin{array}{c}\text { Number of } \\
\text { tested samples }\end{array}$ & $\begin{array}{c}\text { Number of } \\
\text { correct detection }\end{array}$ & $\begin{array}{c}\text { Correct } \\
\text { detection rate } \\
\text { (\%) }\end{array}$ \\
\hline No defect & 80 & 78 & 97.50 \\
\hline Pinholes & 52 & 52 & 100 \\
\hline $\begin{array}{c}\text { Yellow } \\
\text { discoloration }\end{array}$ & 20 & 19 & 95.00 \\
\hline Oil stains & 22 & 21 & 95.45 \\
\hline Scratches & 27 & 25 & 92.59 \\
\hline
\end{tabular}

According to the statistical results in TABLE 1, false detection was found in 2 out of the 80 tested samples with no defect, with a false detection rate of $2.5 \%$. 4 out of 70 samples with defects were undetected, with an average undetected rate of $5.72 \%$ among various defects. Therefore, the proposed algorithm was considered suitable for detecting the defect edge on the surface of aluminum foil.

\section{CONCLUSIONS}

In this paper, combining local fuzzy enhancement, WTMM and multi-scale product, a detection method of surface defects on aluminum foil was proposed. In this method, first, the locations of the defects were calculated in the color space HSI and undergone local fuzzy enhancement, then the method of WTMM and multi-scale product was used to detect the defect edges, including pinholes, yellow discoloration, oil stains and scratches, and the detected defect edges were clear and accurate. In an attempt to verify the effectiveness of the proposed method, it was compared with the detection method based on pre-process of overall fuzzy enhancement, as well as the one that directly adopts WTMM and multi-scale product. Results showed that the proposed method could detect the defect edge better, and thus it could be used as a new approach to detect the defect edges on the surface of aluminum foil.

\section{ACKNOWLEDGEMENT}

This research was supported by the fund project of hzxyjg201528; 2013LX141; 1210006G; 2012PYZR05; KY2016YB454.

\section{REFERENCES}

[1] Jose V A, Fernaado R S. A review of machining monitoring systems based on artificial intelligence process models[J]. Int.J.Adv.Manuf. Technol,2011(47):237-257.

[2] LIN Li-jun, YIN Ying, HE Ming-ge, etc. Edge detection algorithm of magnetic tile crack based on wavelet modulus maxima[J]. Journal of 
University of Electronic Science and Technology of China, 2015,44(2):283-287.

[3] ZHAO Jiuliang, YAN Yunhui, LIU Weiwei, etc. A multi-scale edge detection method of steel strip surface defects online detection system[J]. Journal of Northeastern University(Natural Science), 2010,31(3):432435.

[4] ZHANG Xuewu, DING Yanqiong, DUAN Dunqin, etc. Surface defects inspection of copper strips based on vision bionics[J]. Journal of Image and Graphics, 2011,16(4):593-599.

[5] WANG Xianbao, LI Jie, YAO Minghai, etc. Solar cells surface detection based on deep learning[J]. PR \& AI, 2014,27(6):517-523.

[6] GENG Xin, XIAO Jin, HU Xiaoguang. Color image edge detection and classification based on physical model[J]. Chinese Journal of Scientific Instrument, 2010,31(8):1782-1786.

[7] HUANG Hai-long, WANG Hong, JI Li. Order morphology edge detection algorithm based on partial fuzzy enhancement[J]. Chinese Journal of Scientific Instrument, 2012,33(11):2608-2614.

[8] ISABELLE B. Duality vs. Adjunction for fuzzy mathematical morphology and general form of fuzzy erosions and dilations[J]. Fuzzy Sets and Systems, 2009, 160:1858-1867.

[9] YANG Yongmin, FAN Jizhuang, ZHAO Jie. Steel strip surface detect segmentation based on excess entropy and fuzzy set theory[J]. Optics and Precision Engineering, 2011,19(7):1651-1658.

[10] SHI SHEN, ZHANG YANNING, HU YUENING. A wavelet-based image edge detection and estimation method with adaptive scale selection[J]. International Journal of Wavelets Multiresolution and Information Processing, 2010,8:385-405.

[11] Zhang D, Bao P. A wavelet-based edge detection method by scale multiplication [C] //IEEE 16th International Conference on Pattern Recognition,2002,3:501-504.

[12] Tu C L, Hwang W L, Ho J. Analysis of singularities form modulus maxima of complex wavelets[J]. IEEE Transactions onInformation Theory,2005,51(3): 1049-10 